EGU2020-10236

https://doi.org/10.5194/egusphere-egu2020-10236

EGU General Assembly 2020

(c) Author(s) 2020. This work is distributed under

the Creative Commons Attribution 4.0 License.

\title{
An investigation into the controls on fracture tortuosity in rock sequences and the impact on fluid flow in the upper crust
}

\author{
Nathaniel Forbes Inskip ${ }^{1}$, Tomos Phillips ${ }^{1}$, Kevin Bisdom², Georgy Borisochev ${ }^{1}$, Andreas Busch¹, \\ and Sabine den Hartog ${ }^{1}$ \\ ${ }^{1}$ The Lyell Centre, Institute of GeoEnergy Engineering, Heriot-Watt University, Edinburgh, UK (n.forbes_inskip@hw.ac.uk) \\ ${ }^{2}$ Shell Global Solutions International, Amsterdam, The Netherlands
}

Fractures are ubiquitous in geological sequences, and play an important role in the movement of fluids in the earth's crust, particularly in fields such as hydrogeology, petroleum geology and volcanology. When predicting or analysing fluid flow, fractures are often simplified as a set of smooth parallel plates. In reality, they exhibit tortuosity on a number of scales: Fine-scale tortuosity, or roughness, is the product of the small-scale $(\mu \mathrm{m}-\mathrm{mm})$ irregularities in the fracture surface, whereas large-scale ( $>\mathrm{mm}$ ) tortuosity occurs as a result of anisotropy and heterogeneity within the host formation that leads to the formation of irregularities in the fracture surfaces. It is important to consider such tortuosity when analysing processes that rely on the movement (or hindrance) of fluids flowing through fractures in the subsurface. Such processes include fluid injection into granitic plutons for the extraction of heat in Engineered Geothermal Systems, or the injection of $\mathrm{CO}_{2}$ into reservoirs overlain by fine-grained mudrocks acting as seals in Carbon Capture and Storage projects.

Although it is generally assumed that tortuosity is controlled by factors such as grain size, mineralogy and fracture mode, a systematic study of how these factors quantitatively affect tortuosity is currently lacking. Furthermore, in anisotropic rocks the fracture orientation with respect to any inherent anisotropy is also likely to affect tortuosity.

In order to address this gap, we have induced fractures in a selection of different rock types (mudrocks, sandstones and carbonates) using the Brazil disk method, and imaged the fracture surfaces using both a digital optical microscope and X-ray Computed Tomography. Using these methods we are able to characterise both the fine-scale (roughness) and large-scale tortuosity. In order to understand the effect of fracture orientation on tortuosity we have also analysed fractures induced at different angles to bedding in samples of a highly anisotropic mudrock taken from South Wales, UK. Results indicate that fine-scale tortuosity is highly dependent on the fracture orientation with regards to the bedding plane, with fractures normal to bedding being rougher than those induced parallel to bedding. Finally, in order to measure the effect of tortuosity on fluid flow, we have carried out a series of core flooding experiments on a subset of fractured samples showing that fracture transmissivity decreases with increasing tortuosity. 